\title{
Thoracic outlet syndrome for thoracic surgeons
}

Bryan M. Burt, MD

From the Division of General Thoracic Surgery, Department of Surgery, Baylor College of Medicine, Houston, Tex.

Funding Sources: None.

Received for publication Jan 16, 2018; revisions received Feb 6, 2018; accepted for publication Feb 11, 2018; available ahead of print April 5, 2018.

Address for reprints: Bryan M. Burt, MD, Division of General Thoracic Surgery, Department of Surgery, Baylor College of Medicine, One Baylor Plaza, BCM 390, Houston, TX 77005 (E-mail: bryan.burt@bcm.edu).

J Thorac Cardiovasc Surg 2018;156:1318-23

$0022-5223 / \$ 36.00$

Copyright (c) 2018 by The American Association for Thoracic Surgery

https://doi.org/10.1016/j.jtcvs.2018.02.096

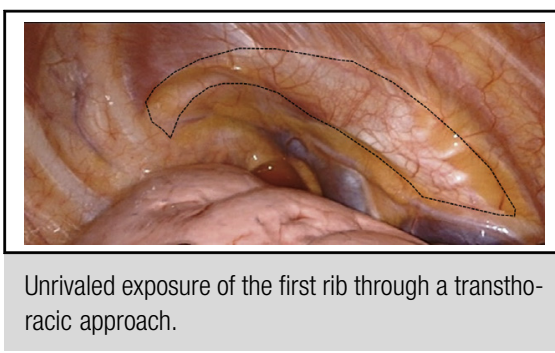

Central Message

A multidisciplinary program benefits patients with TOS. A minimally invasive transthoracic approach provides unparalleled exposure of the first rib and is hypothesized to decrease operative risk.

See Editorial Commentary page 1324. $\checkmark$ Video clip is available online.

Thoracic outlet syndrome (TOS) encompasses a trio of conditions that result from compression of the neurovascular structures that serve the upper extremity: the brachial plexus (neurogenic TOS), the subclavian vein (venous TOS), and the subclavian artery (arterial TOS), and each syndrome is defined by a distinct presentation. This article will address neurogenic thoracic outlet syndrome, the most prevalent and the most controversial form of TOS, and will highlight opportunities for innovation in this field.

Neurogenic TOS is a commonly debilitating condition caused by dynamic compression of the brachial plexus in its traverse between the first rib and scalene muscles, resulting in pain and paresthesias of the upper extremity. Neurogenic TOS is by far the most common type of TOS, with more than $90 \%$ of all TOS cases in large, single-institution series accounted for by neurogenic TOS. ${ }^{1,2}$ Within a more recent multi-institutional national sample through the American College of Surgeons National Surgical Quality Improvement Program database of 1431 patients undergoing first or cervical rib resection for TOS, $83 \%$ of TOS procedures were performed for neurogenic TOS.

The initial treatment of neurogenic TOS nearly always begins with nonsurgical therapy, considered the standard of care for most cases. It is not uncommon, however, to encounter patients who do not tolerate physical therapy. Nonoperative management of neurogenic TOS includes TOS-specific physical therapy, ergonomic modifications, and pain management. Surgery is offered when symptoms become disabling, and its objective is liberation of the brachial plexus from external compression. It is critical here to recognize the existence of neurogenic pectoralis minor syndrome, which has a similar presentation to neurogenic TOS. In neurogenic pectoralis minor syndrome, the anatomic site of brachial plexus compression is the retropectoral space. Pectoralis minor tenotomy is an effective treatment for this condition and can be performed as a low-risk outpatient procedure. ${ }^{4}$ On the basis of physical examination findings and response to lidocaine muscle blocks, neurogenic TOS can be differentiated from neurogenic pectoralis minor syndrome, although some patients will have compression at both sites ("double crush syndrome"). This article will focus on the more prevalent neurogenic TOS, for which resection of the first rib, with either scalenectomy or scalenotomy, is the surgical procedure best suited to decompress the thoracic outlet.

\section{DIAGNOSIS}

Controversy surrounding the field of neurogenic TOS surgery is fueled by variability in both its diagnosis and management, as well as by its poorly understood pathophysiology. Neurogenic TOS is a clinical diagnosis that often presents with nonspecific symptoms that overlap with other clinical disorders, and it is only rarely associated with objective findings. Electromyographic results suggestive of neurogenic TOS or hand atrophy are found only in about $1 \%$ of those with the syndrome, and a cervical rib is present in only $5 \%$ to $9 \%$ of cases of neurogenic TOS. ${ }^{5,6}$ The frequent diagnostic uncertainty in diagnosis is reflected in a 
now-retired nomenclature system for neurogenic TOS that incorporated "disputed TOS" as a clinical entity.

Because of the longstanding absence of well-defined and consistently applied diagnostic criteria for neurogenic TOS, the Society of Vascular Surgery published reporting standards for neurogenic TOS in September 2016. ${ }^{7}$ In this document, diagnostic criteria for neurogenic TOS are proposed to include 3 of the 4 following criteria:

1. Local findings: A history of symptoms consistent with irritation or inflammation at the scalene triangle (pain at the chest wall, axilla, upper back, shoulder, trapezius, neck, or head) and examination findings including pain on palpation of the scalene muscle.

2. Peripheral findings: A history of arm or hand symptoms consistent with central nerve compression (numbness, pain, paresthesias, vasomotor changes, weakness, muscle wasting) and examination findings in which these peripheral symptoms are reproduced by palpation of the scalene muscles or by provocative maneuvers.

3. Absence of other reasonably likely diagnoses, including cervical disk disease, shoulder disease, carpal tunnel disease, and chronic regional pain syndrome.

4. Response to a scalene muscle block.

Although it may not be immediately evident, these proposed diagnostic criteria represent a major advance in the field of neurogenic TOS. Such standardization of diagnostic criteria will improve identification of patients with this disorder and result in use of consistent terms among caregivers, and therefore ultimately has promise to improve patient outcomes. Validation studies will be required and are anticipated to correlate response to treatment with accuracy of the diagnosis made with the proposed criteria.

\section{OUTCOMES REPORTING}

The neurogenic TOS surgical literature is composed almost entirely of retrospective studies, and analyses of symptom improvement are based almost exclusively on subjective measures. Beginning in the 1960s, the first approximately 4 decades of neurogenic TOS publications reported surgical outcomes that used clinical assessment systems with results derived from surgeon and / or patient interpretations of surgical outcome, such as "excellent, good, fair, or poor" or "success, recurrence, or failure." Overall, mostly "excellent" or "good" outcomes were reported for first rib resection for neurogenic TOS. As an example, Urschel and Razzuk ${ }^{8}$ reported "excellent" results in more than $95 \%$ of 2200 transaxillary first rib resections for neurogenic TOS.

It has only been within approximately the last 2 decades that investigators of neurogenic TOS have begun to evaluate surgical outcomes with semiquantitative patient questionnaires of pain and functional disability, including the Disabilities of the Arm, Shoulder and Hand questionnaire, the
Cervical-Brachial Symptom Questionnaire, and the ShortForm 12, among other validated instruments. With these tools, investigators have demonstrated that both physical and mental quality of life improve immediately after surgical decompression for $\operatorname{TOS}^{9}$ and that such quality of life gains are sustained for relatively long periods. ${ }^{10}$ Whereas these self-assessment metrics are regarded as an improvement from the use of clinical assessment scales, they are also limited by incomplete assessment of surgical outcomes: these questionnaires are highly subjective, variable, and subject to the biases inherent in survey sampling. For example, surgical outcomes may influence a subject's willingness to participate and thus lead to selection bias.

Reporting of objective outcome measures in neurogenic TOS is extremely rare. In a recent report, Thompson and colleagues ${ }^{11}$ studied objective performance outcomes in 10 Major League Baseball pitchers with neurogenic TOS who returned to Major League Baseball after first rib resection and physical therapy. ${ }^{11}$ In this study, Thompson and colleagues $^{11}$ compared highly granular career pitching measurements, including 15 traditional pitching metrics (including earned run average, walks plus hits per inning pitched, and strikeout/walk ratio) and 72 advanced pithing metrics with $\mathrm{PitchF} / \mathrm{x}$ data (including pitch velocity, spin rate, and spin direction) before and after neurogenic TOS treatment. On the pitchers' return to Major League Baseball, $75 \%$ of these metrics were unchanged, $19 \%$ were significantly improved, and only $6 \%$ were significantly decreased. This study showcases how innovative approaches with objective performance metrics can define the success of an approach to neurogenic TOS treatment with high confidence. To apply similar concepts to nonathlete patients with neurogenic TOS, one can envision the use of wearable sensors and machine-learning algorithms for evaluation and comparison of preoperative and postoperative upper extremity function as an objective means of assessing response to TOS surgery.

\section{MITIGATION OF RISK BYA MULTIDISCIPLINARY PROGRAM}

Injury precedes neurogenic TOS in approximately 50\% of cases, and some patients may be concurrently seeking economic or legal redress for their injuries. ${ }^{12}$ Notably, these pursuits may interfere with the accuracy of symptom selfreporting. The prevalence of malpractice litigation in neurogenic TOS practice is information that is not readily available to the public; however, neurogenic TOS is often discussed as a surgical discipline associated with substantial legal risk. A review of malpractice lawyer group websites specializing in TOS cases has revealed 2 prominent themes: insufficient nonoperative therapy before surgery and inadequate informed consent, remarked in statements such as, "If your surgeon operated too early or failed to inform you of the risks of surgery and alternatives to surgery, then a 


\section{MULTIDISCIPLINARY TEAM}

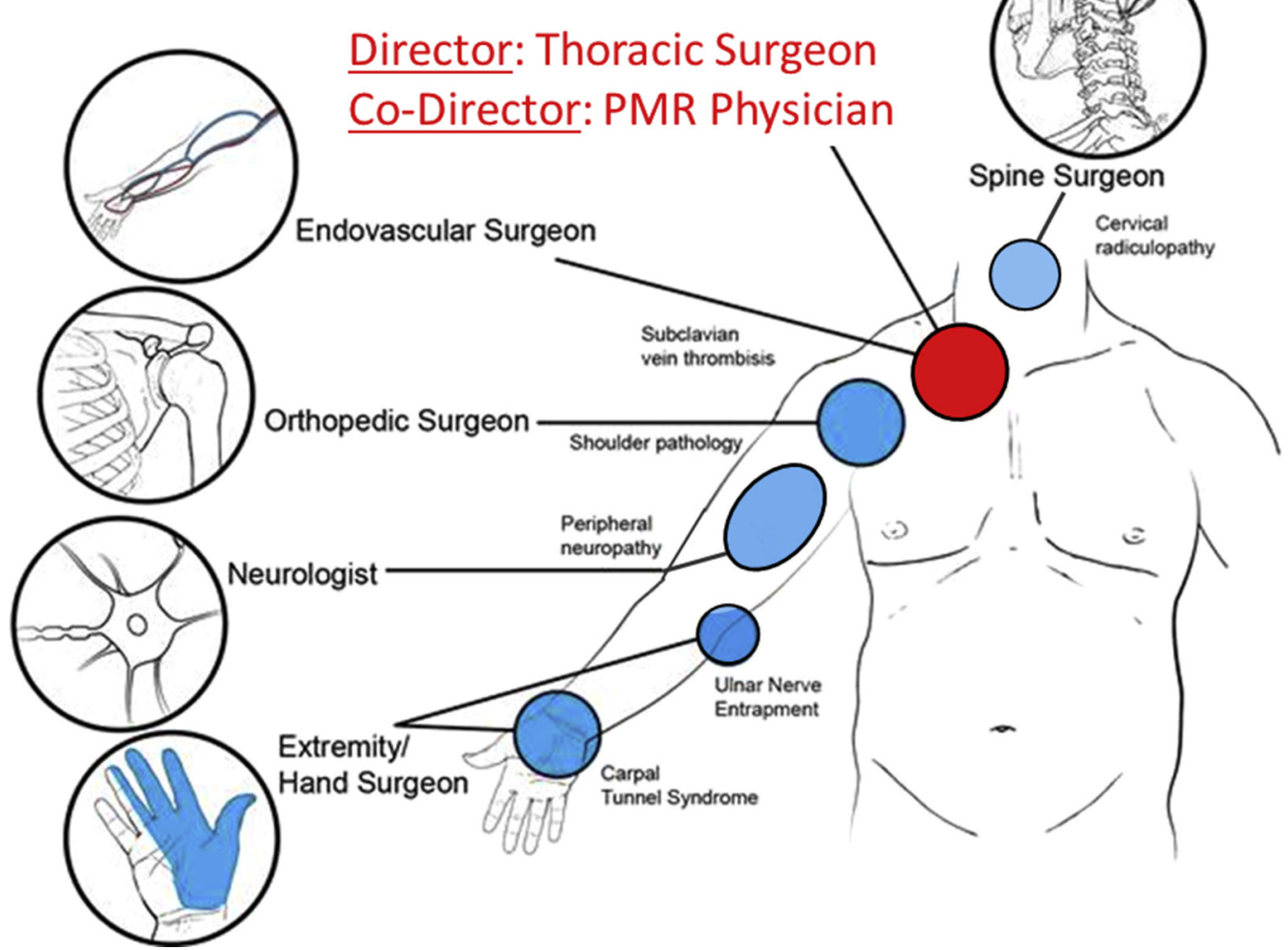

FIGURE 1. A model for a multidisciplinary team approach to thoracic outlet syndrome. $P M R$, Physical medicine and rehabilitation. (Published with permission from the Michael E. DeBakey Department of Surgery at Baylor College of Medicine Houston, Tex.)

lawsuit may be appropriate." In his 1982 presidential address to The American Association for Thoracic Surgery, "The Crisis of Excellence," Dr Thomas B. Ferguson addressed the issue of malpractice in the field of thoracic surgery. ${ }^{13}$ Through access to the National Association of Insurance Commissioners database, Dr Ferguson evaluated malpractice claims involving thoracic surgery between 1975 and 1978 and demonstrated that the greatest frequency of suits $(21 \%)$ occurred after transaxillary first rib resection. Surgical complications were the reasons for these claims, and the most common complication was nerve injury, presumably including brachial plexus injury.

The true incidence of complications from first rib resection, such as brachial plexus injury, is difficult to discern. An anonymous national survey published in 1982 demonstrated that $52 \%$ of the 259 participating surgeons performing transaxillary first rib resection observed brachial plexus injury resulting in complete postoperative paralysis in 102 instances (33 from personal cases and 69 observed among colleagues) and resulting in partial neurologic deficit in 171 instances (60 personal cases and 111 observed cases). ${ }^{14}$ In contrast, in published large, singleinstitution series of first rib resection, the rate of brachial plexus injury from first rib resection ranges $0 \%^{2,6,15-17}$ to $0.08 \%{ }^{18}$ to $1.1 \%{ }^{19}$ Similarly, a multi-institution administrative database study evaluating 2016 first rib resections for neurogenic TOS reported that brachial plexus injury occurs in $0.6 \%$ of first rib resections. ${ }^{20}$ In a relatively smaller single-institution series of 102 first rib resections, however, brachial plexus injury was reported in $9 \%$ of first rib resections performed through the transaxillary approach and $4 \%$ of first rib resections performed through the supraclavicular approach. ${ }^{21}$ Additional technical complications resultant from first rib resection include phrenic nerve injury, long thoracic nerve injury, and injury to the subclavian vessels. Taken together, it is reasonable to conclude that publication biases limit our understanding of the true incidence of complications after first rib resection.

Multidisciplinary team approaches have been conclusively shown to improve quality of life and long-term outcomes in patients with a variety of cancers, including those treated surgically (a representation is given in reference $^{22}$ ). At my institution (Baylor College of Medicine, Houston, Tex), we believe that the same principles can be applied to patients with neurogenic 


\begin{tabular}{|c|c|c|c|}
\hline New Office Visit & Diagnostics & Therapy & Follow-up \\
\hline \multirow{4}{*}{$\begin{array}{c}\text { Thoracic Surgeon } \\
+\end{array}$} & Physical Exam & & \\
\hline & C-spine $x$-ray & TOS targeted PT & \\
\hline & C-snine MRI & \multirow{3}{*}{ 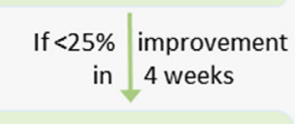 } & 2 weeks \\
\hline & C-spine IVIRI & & 2 months \\
\hline PMR Physician & EMG & & \multirow{2}{*}{6 months } \\
\hline \multirow{5}{*}{$\begin{array}{c}\text { Symptom } \\
\text { Questionnaires }\end{array}$} & Muscle block & \multirow{2}{*}{ First Rib Resection } & \\
\hline & \pm & & yearly \\
\hline & Ultrasound & \multirow{3}{*}{$\begin{array}{c}\text { OR } \\
\text { Pec Minor Release }\end{array}$} & \\
\hline & \pm & & $\begin{array}{l}\text { Symptom } \\
\text { Questionnaires }\end{array}$ \\
\hline & Specialist Consult & & \\
\hline
\end{tabular}

FIGURE 2. The Baylor College of Medicine thoracic surgery diagnostic and therapeutic algorithm for neurogenic thoracic outlet syndrome (TOS). Every new patient with suspected neurogenic thoracic outlet syndrome is seen by a thoracic surgeon and a physical medicine and rehabilitation $(P M R)$ physician. A cervical spine (C-spine) radiograph is performed to identify the presence of cervical ribs. A cervical spine magnetic resonance imaging $(M R I)$ scan is performed to evaluate the presence of radiculopathy; if results are abnormal, the patient is evaluated by a spine surgeon. Electromyography $(E M G)$ is performed to rule out ulnar nerve entrapment and carpal tunnel syndrome; if either is present, the patient is evaluated by an extremity surgeon. Physical examination and muscle blocks are performed to determine the predominant site of compression of the brachial plexus at either the scalene triangle or retropectoral space. Vascular ultrasonography performed if elements of venous or arterial thoracic outlet syndrome are present. PT, Physical therapy; Pec Minor, pectoralis minor.

TOS, and that a structured multidisciplinary strategy for neurogenic TOS (Figure 1) will (1) mitigate legal risk and (2) improve patient outcomes. The proposed benefits of such a program include increase in diagnostic accuracy for neurogenic TOS and for competing diagnoses, decrease in time to definitive treatment, establishment of clinical protocols to streamline and standardize care (Figure 2), and assurance of appropriate "standard-ofcare" treatment algorithms. Integrated into this program are mechanisms to guarantee and document comprehensive informed consent before any surgical procedure (Figure E1). A model that we have found particularly useful is one that includes the thoracic surgeon and a physical medicine and rehabilitation physician in leadership roles. In this system, every new patient with suspected neurogenic TOS is evaluated by both these physicians on their initial visit, and all new and established cases are reviewed weekly by these 2 core team members. The physical medicine and rehabilitation component of this program has proved indispensable for refinement of diagnosis, for arrangement and evaluation of response to physical therapy, and for management of chronic pain and disability. Other critical members of the team include a vascular or endovascular surgeon for collaboration on venous and arterial TOS cases, a spine surgeon for evaluation of cervical radiculopathy when appropriate, and shoulder and extremity surgeons for cases including possible shoulder pathology, ulnar nerve entrapment, and carpal tunnel syndrome. Further, because symptoms of neurogenic TOS may be associated with complex psychologic problems, including somatoform disorders, ${ }^{23}$ recruitment of a psychiatrist to the program may have substantial value.

\section{TECHNIQUE}

Thoracic outlet syndrome is a condition that is treated surgically by a variety of surgical subspecialties including thoracic surgery, vascular surgery, orthopedic surgery, neurosurgery, and plastic surgery. Among the 1431 first/cervical rib resection sample from the American College of Surgeons National Surgical Quality Improvement Program database, it was determined that $90 \%$ of procedures were performed by vascular surgeons (3), however, thoracic surgeons are uniquely positioned to care for patients with thoracic outlet syndrome.

Resection of the first rib with either scalenectomy or scalenotomy is procedure required to decompress the thoracic outlet in neurogenic TOS. It is often discussed that complete resection of the posterior first rib (to the level of the transverse process) is necessary to achieve favorable surgical outcome in neurogenic TOS. On the basis of the anatomy of the thoracic outlet and studies that correlate a long stump of residual rib after rib resection with early recurrence of neurogenic $\mathrm{TOS},{ }^{24}$ this is a rationally grounded principle.

The most common approaches to first rib resection are supraclavicular and transaxillary. Large published experiences with each approach have demonstrated good outcomes, and the superiority of one approach to the other 

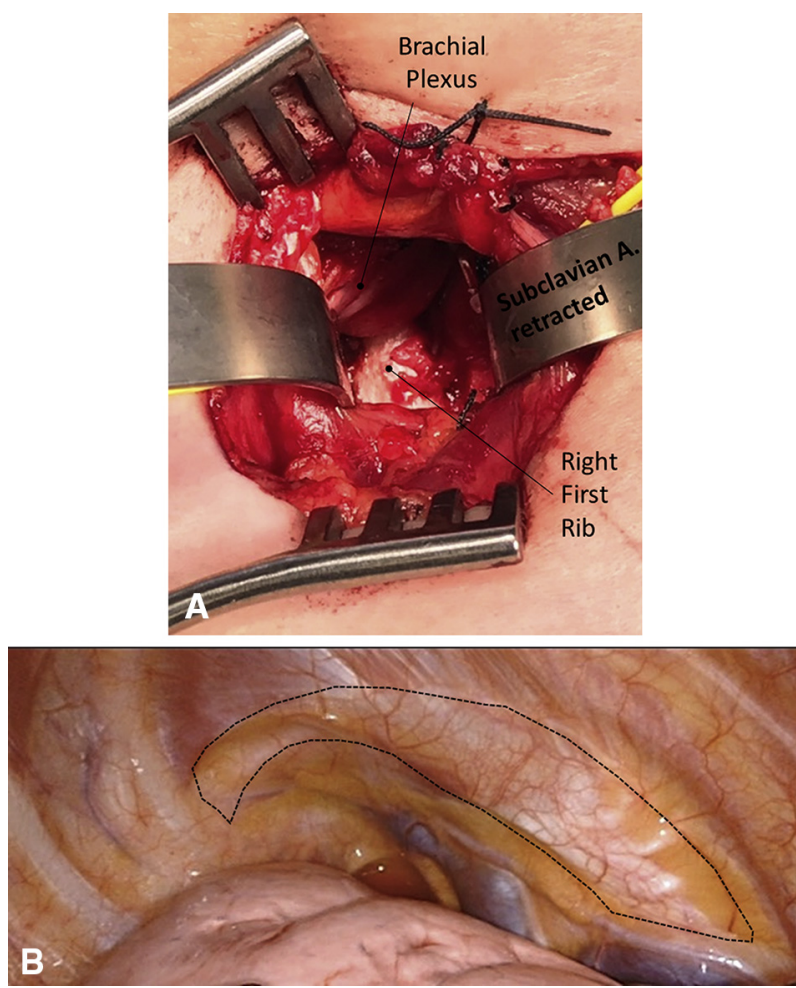

FIGURE 3. A, Exposure of the right first rib by the supraclavicular approach. Note that a small fraction of the first rib is visible following retraction of the brachial plexus and the subclavian artery. B, Unrivaled exposure of the entire right first rib is provided by a right robotically assisted thoracoscopic approach, requiring no retraction of neurovascular structures.

has not been definitively demonstrated. Both approaches, however, share common disadvantages. Shortcomings of both the supraclavicular and transaxillary approaches include limited exposure and visualization of the first rib and a requirement for manipulation or retraction of the brachial plexus, the latter of which may be more pronounced during supraclavicular first rib resection. Moreover, these disadvantages are amplified in patients with overweight body habitus during each of these approaches. Exposure of the right first rib through the supraclavicular approach is illustrated in Figure 3, A, and limited exposure of the first rib is also present in the transaxillary approach.

Transthoracic approaches to first rib resection have been reported and include thoracoscopic transthoracic first rib resection ${ }^{25}$ and thoracoscopically assisted transaxillary first rib resection. ${ }^{26}$ Robotically assisted thoracoscopic first rib resection has also been described. ${ }^{27,28} \mathrm{~A}$ minimally invasive (robotic or thoracoscopic) transthoracic approach to first rib resection has clear advantages relative to traditional approaches, namely (1) unrivaled visualization of the entire (anterior and posterior) first rib (Figure 3,B), (2) no manipulation (retraction or stretching) of the brachial

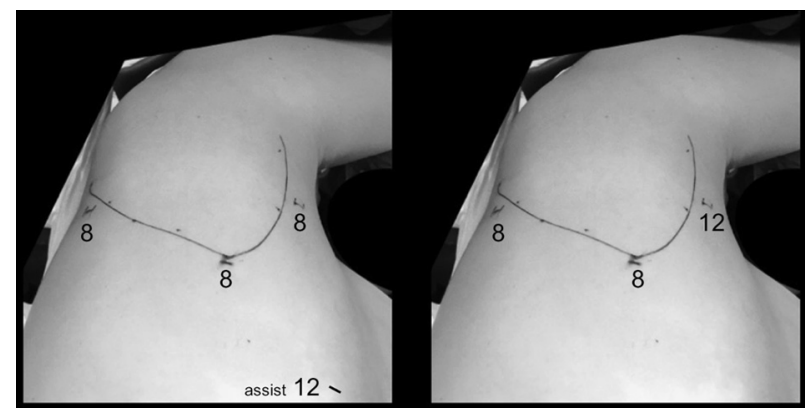

VIDEO 1. A video of B.M.B.'s technique of robotically assisted thoracoscopic first rib resection for neurogenic and venous thoracic outlet syndrome (TOS). I.V., Intravenous; SVC, superior vena cava; Phrenic $N$., phrenic nerve. Video available at: http://www.jtcvsonline.org/article/ S0022-5223(18)30778-5/fulltext.

plexus or subclavian vessels, and (3) independence from body habitus in attaining consistent outstanding exposure. These attributes of this approach, taken together with sustained visualization of the brachial plexus, subclavian artery, subclavian vein, and phrenic nerve, can be rationally predicted to mitigate complications from first rib resection. An additional advantage of the unprecedented and videoprojected exposure afforded by a transthoracic minimally invasive approach is facilitation of the teaching of residents, fellows, and all members of the operating room team. A video of my technique of robotically assisted thoracoscopic first rib resection for neurogenic TOS (and also for venous TOS) accompanies this article (Video 1).

\section{CONCLUSIONS}

Many of the controversies that surround neurogenic TOS are opportunities for innovation. The great majority of TOS cases are neurogenic, and thoracic surgeons are uniquely equipped to care for these patients. The minimally invasive transthoracic approach to first rib resection provides unrivaled exposure to the first rib, regardless of patient build, without need for manipulation of the brachial plexus or subclavian vessels, and it is expected to minimize operative risk.

\section{Conflict of Interest Statement}

Author has nothing to disclose with regard to commercial support.

I thank Scott Holmes, a member of the Michael E. DeBakey Department of Surgery at Baylor College of Medicine, for his illustrative assistance during the preparation of this article, and Ellie Biaghoshi for administrative assistance with manuscript preparation.

\section{References}

1. Sanders RJ, Hammond SL, Rao NM. Thoracic outlet syndrome: a review. Neurologist. 2008;14:365-73. 
2. Hempel GK, Shutze WP, Anderson JF, Bukhari HI. 770 consecutive supraclavicular first rib resections for thoracic outlet syndrome. Ann Vasc Surg. 1996;10:456-63.

3. Rinehardt EK, Scarborough JE, Bennett KM. Current practice of thoracic outlet decompression surgery in the United States. J Vasc Surg. 2017;66:858-65.

4. Vemuri C, Wittenberg AM, Caputo FJ, Earley JA, Driskill MR, Rastogi R, et al. Early effectiveness of isolated pectoralis minor tenotomy in selected patients with neurogenic thoracic outlet syndrome. J Vasc Surg. 2013;57:1345-52.

5. Sanders RJ, Hammond SL, Rao NM. Diagnosis of thoracic outlet syndrome. J Vasc Surg. 2007;46:601-4.

6. Orlando MS, Likes KC, Mirza S, Cao Y, Cohen A, Lum YW, et al. A decade of excellent outcomes after surgical intervention in 538 patients with thoracic outlet syndrome. J Am Coll Surg. 2015;220:934-9.

7. Illig KA, Donahue D, Duncan A, Freischlag J, Gelabert H, Johansen K, et al. Reporting standards of the Society for Vascular Surgery for thoracic outlet syndrome. J Vasc Surg. 2016;64:e23-35.

8. Urschel HC Jr, Razzuk MA. Upper plexus thoracic outlet syndrome: optimal therapy. Ann Thorac Surg. 1997;63:935-9.

9. Chang DC, Rotellini-Coltvet LA, Mukherjee D, De Leon R, Freischlag JA. Surgical intervention for thoracic outlet syndrome improves patient's quality of life. J Vasc Surg. 2009;49:630-5; discussion 5-7.

10. Rochlin DH, Gilson MM, Likes KC, Graf E, Ford N, Christo PJ, et al. Quality-oflife scores in neurogenic thoracic outlet syndrome patients undergoing first rib resection and scalenectomy. J Vasc Surg. 2013;57:436-43.

11. Thompson RW, Dawkins C, Vemuri C, Mulholland MW, Hadzinsky TD, Pearl GJ. Performance metrics in professional baseball pitchers before and after surgical treatment for neurogenic thoracic outlet syndrome. Ann Vasc Surg. 2017; 39:216-27.

12. Oates SD, Daley RA. Thoracic outlet syndrome. Hand Clin. 1996;12:705-18.

13. Ferguson TB. The crisis of excellence. J Thorac Cardiovasc Surg. 1982;84:161-71.

14. Dale WA. Thoracic outlet compression syndrome. Critique in 1982. Arch Surg. 1982;117:1437-45.

15. Jamieson WG, Chinnick B. Thoracic outlet syndrome: fact or fancy? A review of 409 consecutive patients who underwent operation. Can J Surg. 1996;39:321-6.

16. Axelrod DA, Proctor MC, Geisser ME, Roth RS, Greenfield LJ. Outcomes after surgery for thoracic outlet syndrome. J Vasc Surg. 2001;33:1220-5.
17. Caputo FJ, Wittenberg AM, Vemuri C, Driskill MR, Earley JA, Rastogi R, et al Supraclavicular decompression for neurogenic thoracic outlet syndrome in adolescent and adult populations. J Vasc Surg. 2013;57:149-57.

18. Urschel HC Jr. Neurovascular compression in the thoracic outlet: changing management over 50 years. Adv Surg.

19. Degeorges R, Reynaud C, Becquemin JP. Thoracic outlet syndrome surgery: long-term functional results. Ann Vasc Surg. 2004;18:558-65.

20. Chang DC, Lidor AO, Matsen SL, Freischlag JA. Reported in-hospital complications following rib resections for neurogenic thoracic outlet syndrome. Ann Vasc Surg. 2007;21:564-70.

21. Hosseinian MA, Loron AG, Soleimanifard Y. Evaluation of complications after surgical treatment of thoracic outlet syndrome. Korean J Thorac Cardiovasc Surg. 2017;50:36-40

22. Tamburini N, Maniscalco P, Mazzara S, Maietti E, Santini A, Calia N et al. Multidisciplinary management improves survival at 1 year after surgical treatment for non-small-cell lung cancer: a propensity score-matched study. Eur J Cardiothorac Surg. December 25, 2017 [Epub ahead of print].

23. Hameed SM, Grewal RP, Urschel JD. Somatization tendencies in surgically treated neurogenic thoracic outlet syndrome patients. Vasc Endovasc Surg 1995;29:184-8.

24. Mingoli A, Feldhaus RJ, Farina C, Cavallari N, Sapienza P, di Marzo L, et al Long-term outcome after transaxillary approach for thoracic outlet syndrome. Surgery. 1995;118:840-4.

25. George RS, Milton R, Chaudhuri N, Kefaloyannis E, Papagiannopoulos K Totally Endoscopic (VATS) first rib resection for thoracic outlet syndrome. Ann Thorac Surg. 2017;103:241-5.

26. Soukiasian HJ, Shouhed D, Serna-Gallgos D, McKenna R III, Bairamian VJ, McKenna RJ Jr. A video-assisted thoracoscopic approach to transaxillary first rib resection. Innovations. 2015;10:21-6.

27. Strother EM, Margolis M. Robotic first rib resection. Oper Tech Thorac Cardiovasc Surg. 2015;20:176-88.

28. Gharagozloo F, Meyer M, Tempesta BJ, Margolis M, Strother ET, Tummala S. Robotic en bloc first-rib resection for Paget-Schroetter disease, a form of thoracic outlet syndrome: technique and initial results. Innovations. 2012;7: 39-44. 


\section{Thoracic Surgery Visit and Procedure Patient Information}

I acknowledge that I have become a patient of I am scheduled for

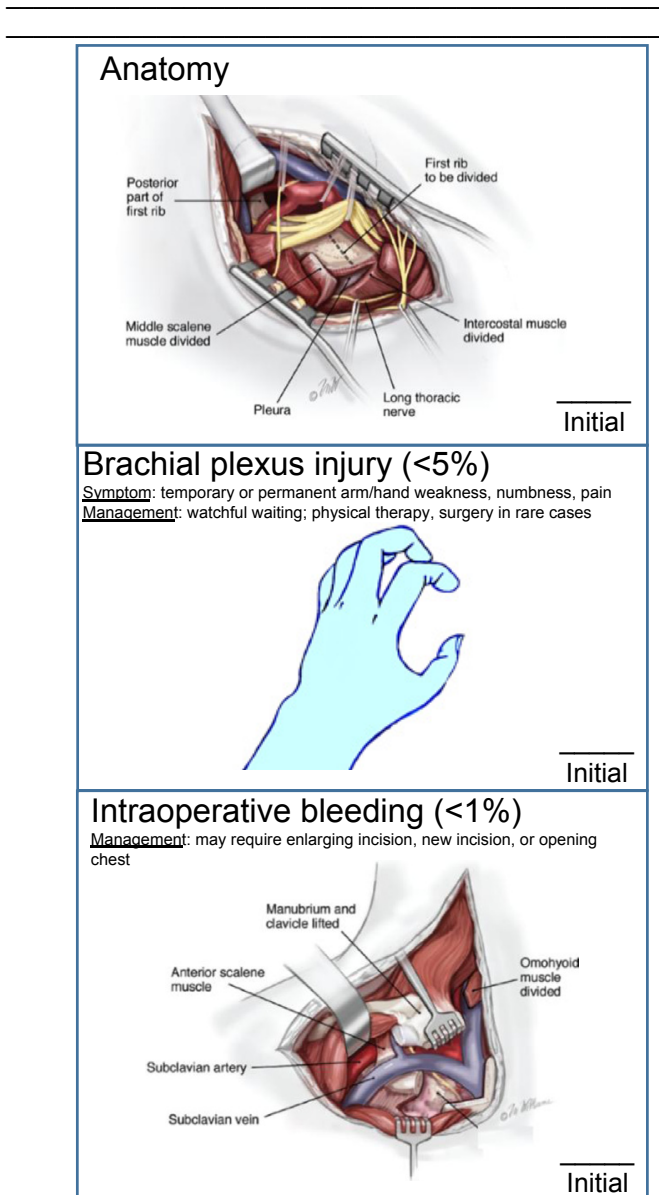

in the Division of Thoracic Surgery and

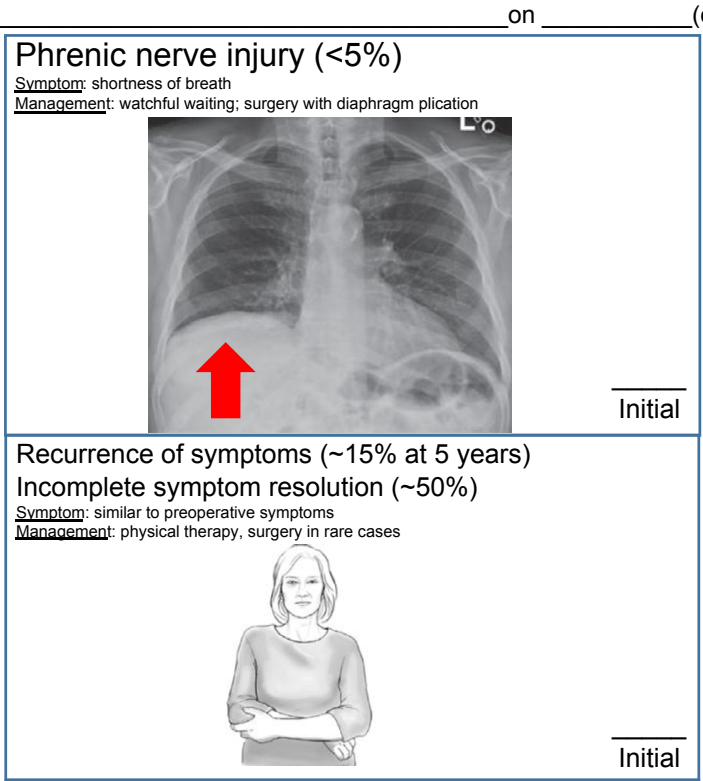

\section{Chyle leak $(<5 \%)$}

Symptom: neck swelling, milky discharge from drain or neck

Management: low fat diet, pressure dressing, reoperation in neck or chest

date).

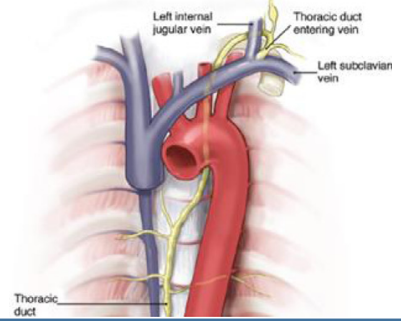

Initial

I understand that the above list does not include all possible complications or outcomes as a result of this procedure or hospitalization and that it is not possible for my doctor to predict whether these complications, known or unknown, may occur. My doctor has told me that the estimated risk of dying from this procedure is $\%$.

My doctor has explained that the Thoracic Surgery Team includes surgeons, residents (physicians-in-training), fellows, nurses, nurse practitioners and physician assistants, under the direction of my doctor and these individuals will participate in all stages of my care and treatment.

All of my questions and the questions of my family regarding the potential risks and benefits of the recommended procedure ha ve been answered. If I have further questions or concerns, I know that I can contact my doctor's office and request a meeting toaddress these concerns. I am now requesting to be scheduled for surgery.

Date Time Patient Signature

Date Time Physician $\mathrm{MD}$

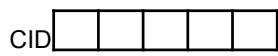

THIS DOCUMENT IS NOT A CONSENT FOR PROCEDURE

FIGURE E1. Possible format of consent form. (Reprinted with permission from McGraw Hill.) 